Research Article

\title{
Simultaneous Determination of Malachite Green, Chloramphenicols, Sulfonamides, and Fluoroquinolones Residues in Fish by Liquid Chromatography-Mass Spectrometry
}

\author{
Yongping Chen $\mathbb{D}^{\mathbb{D}},{ }^{1}$ Sudong Xia $\mathbb{D}^{\mathrm{D}},{ }^{2}$ Xianqin Han $\mathbb{D},{ }^{1}$ and Zhiru Fu $\mathbb{D}^{1}$ \\ ${ }^{1}$ Tianjin Agricultural Ecological Environment Monitoring and Agricultural Product Quality Testing Center, \\ Tianjin 300221, China \\ ${ }^{2}$ Tianjin Key Lab of Aqua-Ecology and Aquaculture, Department of Fishery Science, Tianjin Agricultural University, \\ Tianjin 300384, China
}

Correspondence should be addressed to Zhiru Fu; zhfu117@163.com

Received 24 April 2019; Revised 27 December 2019; Accepted 14 January 2020; Published 19 February 2020

Academic Editor: Luca Campone

Copyright (c) 2020 Yongping Chen et al. This is an open access article distributed under the Creative Commons Attribution License, which permits unrestricted use, distribution, and reproduction in any medium, provided the original work is properly cited.

\begin{abstract}
A fast-analytical method using simplified extraction has been developed for the simultaneous determination of 42 compounds from 4 different classes of veterinary drugs (amphenicols, triphenylmethane, fluoroquinolones, and sulfonamides) in fish by reverse phase liquid chromatography-tandem mass spectrometry. The selection of extraction reagents was optimized using different types of microfiltration membrane, mobile phase, and LC column. Samples were extracted using $0.4 \%$ hydrochloric acid in acetonitrile and ethyl acetate and then were cleaned up using solid-phase extraction Cleanert Alumina N columns (500 mg) and Oasis hydrophiliclipophilic balance (HLB) cartridges. The chromatographic separation was performed on a XR-ODS $\mathrm{C}_{8}$ column using a mobile phase of (A) $0.1 \%$ formic acid and $2 \mathrm{mM}$ ammonium acetate and (B) $0.1 \%$ formic acid acetonitrile at a flow rate of $0.25 \mathrm{~mL} \cdot \mathrm{min}^{-1}$. The results indicated $67.7-112.8 \%$ recovery of 42 compounds with an intra- and interday relative standard deviations less than $10 \%$. The limits of quantification for analytes were in the range of $0.3-1.0 \mu \mathrm{g} \mathrm{kg}^{-1}$ for samples which were satisfactory to support future surveillance monitoring. The method applicability was checked by analyzing 30 fish samples collected from local markets. Two fish samples surpassed the established MRL of $100 \mu \mathrm{g} \mathrm{kg}^{-1}$ with values of $104 \mu \mathrm{g} \mathrm{kg}^{-1}$ and $112 \mu \mathrm{g} \mathrm{kg}^{-1}$.
\end{abstract}

\section{Introduction}

Antibiotics are used widely to treat animal diseases in aquacultures, such as bacterial infectious diseases, and as feed additives to promote the growth of aquaculture animals [1-3]. Beyond that, antibiotics are used extensively in humans and animals to prevent and treat diseases. A large number of antibiotics that have not been metabolized can enter the aquatic environment through direct discharge of animal wastewater or by leaking or running off from manure fertilizer on agricultural land [4]. Eventually, antibiotics can be present in the water and sediment of the living environment of aquaculture animals. Antibiotics in the environment can lead to residuals in aquatic animals $[5,6]$. Chloramphenicol, florfenicol, malachite green, sulfonamides
(SAs), and quinolones (QNs) are effective topical fungicides used in the aquaculture industry [7]. Chloramphenicol, florfenicol, and malachite green are prohibited as aquaculture veterinary drugs in many countries including the United States, Canada, and China and in the European Union. However, these drug residues which settle in aquatic tissues can cause some adverse human health effects, such as allergic reactions and alterations in the delicate balance of intestinal flora, and they can produce resistance to antibiotics [8-14]. Malachite green and its metabolites are toxic, mutagenic, and carcinogenic. A number of studies have been described for the determination of antibiotics, malachite green, and leucomalachite green in different sample treatment methods and instrumental determination methods [15-18]. However, these methods are focused only on single-drug class of veterinary residues, which 
leads to low efficiency and high cost when different physicochemical properties of various veterinary drugs are determined separately. The use of multiresidue methods for the simultaneous determination of different classes of compounds is a widely spreading form of analysis in laboratories that perform official controls, research facilities, or laboratories in the private sector [19]. The use of multiresidue methods is becoming necessary in routine laboratories for the analysis of veterinary drugs (e.g., SAs, QNs, and tetracyclines). Therefore, the research trend is to develop methods which can determine multiclass compounds in a single analytical process by liquid chromatography-tandem mass spectrometry (LC-MS/MS).

The development of simultaneous multiclass drug residue determination is challenging work because concentrations of analyte are low in the tissues of the fish. In addition, there is the inherent complexity of matrices containing high protein and fat content [20]. The analytical procedure normally includes extraction, purification, and instrumental determination of compounds. Extraction and purification are crucial steps to achieve the satisfactory recovery and purifying effect simultaneously for different classes of compounds from fish sample. There are many studies on the determination of sulfonamides (SAs) [21-24], fluoroquinolones (QNs) [25-31], malachite green, and leucomalachite green and their application in food matrices or environmental matrices [32-34]. It is inefficient not to meet the requirement of determining different kinds of compounds in fish sample simultaneously. It is necessary to increase the efficiency and reduce the cost and time of these analyses.

The objective of the present study is to develop and validate an analytical method using LC-MS/MS to simultaneously extract and analyze amphenicols, leucomalachite green, malachite green, QNs, and SAs in fish sample. Analytical performance of the proposed method was evaluated through a validation study which involved assessment of parameters including linearity, specificity, recovery, precision, and limits of detection (LODs) and limits of quantification (LOQs). The developed method was applied to determine the concentration levels of the selected SAs, QNs, chloramphenicol, florfenicol, leucomalachite green, and malachite green in fish sample.

\section{Materials and Methods}

2.1. Materials and Reagents. All solvents were of MS grade. Acetonitrile and ethyl acetate were supplied from Merck (Darmstadt, Germany). Formic acid was purchased from Sigma-Aldrich (Saint Louis, MO, USA). Hydrochloric acid was provided by Kemiou (Tianjin, China). Ammonium acetate was obtained from Sigma-Aldrich. Water was purified using a Milli-Q Synthesis system from Millipore (Bedford, MA, USA). SPE Cleanert Alumina N columns (500 mg) were provided by Agela (Tianjin, China). Oasis HLB cartridges $(6 \mathrm{~mL}, 200 \mathrm{mg}$ or $6 \mathrm{~mL}, 500 \mathrm{mg})$ were supplied by Waters (Milford, MA, USA). Syringe filters (GHP ACRODISC $130.2 \mu \mathrm{m}$ ) were purchased from Pall Corporation (Ann Arbor, MI, USA).

The target standards of sulfisoxazole, sulfisomidine, sulfathiazole, sulfapyridine, sulfamonomethoxine, sulfamethoxypyridazine, sulfamethoxazole, sulfamethizole, sulfamethazine, sulfameter, sulfamerazine, sulfaguanidine, sulfadoxine, sulfadimethoxine, sulfadiazine, sulfachloropyridazine, sulfachinoxalin, sulfabenzamide, sulfachloropyrazine, sulfaphenazole, sulfacetamide, sulfamoxol, fleroxacin, ofloxacin, norfloxacin, enoxacin, ciprofloxacin, enrofloxacin, lomefloxacin, danofloxacin, orbifloxacin, difloxacin, sarafloxacin, sparfloxacin, oxolinic acid, flumequine, pefloxacin, nalidixic acid, chloramphenicol, florfenicol, leucomalachite green and malachite green, sulfadoxine-D3, sulfadimethoxine-D6, norfloxacin-D5, ciprofloxacin-D8, enrofloxacin-D5, chloramphenicol-D5, malachite green-D5, and leucomalachite green-D6 were purchased from Dr. Ehrenstorfer, GmbH (Augsburg, Germany).

About $10 \mathrm{mg}$ of individual standard (corrected by purity and salt form) was accurately weighed. The compounds were dissolved in $10 \mathrm{~mL}$ of acetonitrile. Standard stock solutions $\left(1 \mathrm{mg} \mathrm{mL}^{-1}\right)$ were stored at $-20^{\circ} \mathrm{C}$ and were stable for at least 6 months. Mixed working standard solutions were prepared by diluting the stock solution with acetonitrile. These solutions were stored at $4^{\circ} \mathrm{C}$ and were stable for 3 months.

The ammonium acetate solution was prepared by dissolving $0.08 \mathrm{~g}$ of $\mathrm{CH}_{3} \mathrm{COONH}_{4}$ in a $500 \mathrm{~mL}$ volumetric flask with about $500 \mathrm{~mL}$ of water and aqueous $0.1 \%$ formic acid. Acidified acetonitrile was prepared by mixing $4 \mathrm{~mL}$ of hydrochloric acid and $1 \mathrm{~L}$ acetonitrile. HLB cartridges were prepared by activating with $6 \mathrm{~mL}$ methanol and $6 \mathrm{~mL}$ formic acid-water solution $(\mathrm{pH}=3)$.

\subsection{Extraction and Cleanup of Veterinary Drug Residue.} For the extraction of compounds in fish samples which were obtained from an aquafarm, previously homogenized tissue was weighed $(5.0 \pm 0.02 \mathrm{~g})$ into a $50 \mathrm{~mL}$ polypropylene tube, and $100 \mu \mathrm{L}$ of mixed internal standard working solutions was added to samples (sulfadoxine-D3, sulfadimethoxineD6, norfloxacin-D5, ciprofloxacin-D8, enrofloxacin-D5 $1.0 \mu \mathrm{g} \mathrm{mL}^{-1}$, chloramphenicol-D5, malachite green-D5, and leucomalachite green-D6 $40 \mathrm{ng} \mathrm{mL}^{-1}$ ). $10 \mathrm{~mL}$ ethyl acetate was added to each tube, and then the suspension was homogenized with a T18 basic Ultra Turrax for $2 \mathrm{~min}$. The suspension was sonicated for $25 \mathrm{~min}$ in water bath at $35^{\circ} \mathrm{C}$ and then centrifuged (Sigma 3-18KS, Osterode am Harz, Germany) at $3743 \times g$ for $10 \mathrm{~min}$ at $4^{\circ} \mathrm{C}$. The supernatant was transferred to a $20 \mathrm{~mL}$ glass centrifuge tube. Anhydrous sodium sulfate $(10 \mathrm{~g})$ was added to the residue and then $10 \mathrm{~mL}$ acidified acetonitrile was added to the residue followed by mixing on a vortex mixer for $2 \mathrm{~min}$. All polypropylene tubes were then ultrasonicated for $15 \mathrm{~min}$ and centrifuged at $10,397 \times g$ for $10 \mathrm{~min}$ at $4^{\circ} \mathrm{C}$. The supernatant from each tube was transferred to a $20 \mathrm{~mL}$ glass centrifuge tube combined with the first extraction. The extracted solution from each sample was dried in a water bath at $40^{\circ} \mathrm{C}$ under nitrogen to remove the organic solvent and then dissolved with $5 \mathrm{~mL}$ water. Dissolved sample extract was adjusted to $\mathrm{pH} 3$ [35] using $4 \mathrm{M} \mathrm{H}_{2} \mathrm{SO}_{4}$.

Sample extracts contained a lot of fat and protein, which could cause matrix interference. SPE Cleanert Alumina N 
TABLE 1: Transitions and optimal conditions used for MS/MS analysis.

\begin{tabular}{|c|c|c|c|c|c|}
\hline Analyte & Retention time (min) & $\begin{array}{l}\text { Precursor ion } \\
\quad(m / z)\end{array}$ & $\begin{array}{l}\text { Product ion } \\
(m / z)\end{array}$ & Declustering potential (V) & Collision energy $(\mathrm{eV})$ \\
\hline Fleroxacin & 7.73 & 370 & $269 / 326^{*}$ & 90 & $26 / 19$ \\
\hline Ofloxacin & 7.75 & 362 & $261 / 318^{*}$ & 85 & $27 / 18$ \\
\hline Norfloxacin & 7.75 & 320.3 & $233.4 / 276.3^{*}$ & 85 & $26 / 26$ \\
\hline Enoxacin & 6.79 & 321 & $232 / 303^{*}$ & 85 & $34 / 21$ \\
\hline Ciprofloxacin & 7.33 & 332.2 & $245.3 / 288.3^{*}$ & 85 & $35 / 25$ \\
\hline Enrofloxacin & 8.07 & 360.2 & $245.2 / 316.3^{*}$ & 90 & $39 / 27$ \\
\hline Lomefloxacin & 7.66 & 352 & $265 / 308^{*}$ & 85 & $22 / 16$ \\
\hline Danofloxacin & 7.86 & 358 & $283 / 340^{*}$ & 85 & $22 / 22$ \\
\hline Orbifloxacin & 7.96 & 396 & $295 / 352^{*}$ & 85 & $24 / 17$ \\
\hline Difloxacin & 8.07 & 400 & $299 / 356^{*}$ & 85 & $28 / 19$ \\
\hline Sarafloxacin & 8.05 & 386 & $299 / 342^{*}$ & 85 & $26 / 18$ \\
\hline Sparfloxacin & 9.04 & 393 & $292 / 349^{*}$ & 85 & $25 / 19$ \\
\hline Oxolinic acid & 9.72 & 262 & $216 / 244^{*}$ & 85 & $29 / 18$ \\
\hline Flumequine & 10.07 & 262 & $202 / 244^{*}$ & 85 & $32 / 18$ \\
\hline Pefloxacin & 7.20 & 334 & $290 / 316^{*}$ & 85 & $18 / 20$ \\
\hline Nalidixic acid & 10.01 & 233.3 & $187.4 / 215.2^{*}$ & 85 & $36 / 24$ \\
\hline Sulfisoxazole & 9.49 & 268 & $108 / 156^{*}$ & 85 & $22 / 13$ \\
\hline Sulfisomidine & 7.05 & 279 & $186 / 156^{*}$ & 85 & $17 / 19$ \\
\hline Sulfathiazole & 4.65 & 256 & $108 / 156^{*}$ & 85 & $22 / 16$ \\
\hline Sulfapyridine & 4.98 & 250 & $184 / 156^{*}$ & 85 & $18 / 16$ \\
\hline Sulfamonomethoxine & 8.44 & 281 & $215 / 156^{*}$ & 85 & $17 / 17$ \\
\hline Fleroxacin & 7.49 & 281 & $215 / 156^{*}$ & 85 & $17 / 17$ \\
\hline Ofloxacin & 6.90 & 254 & $108 / 156^{*}$ & 85 & $22 / 16$ \\
\hline Norfloxacin & 7.21 & 271 & $107 / 156^{*}$ & 85 & $30 / 14$ \\
\hline Enoxacin & 3.26 & 279 & $186 / 156^{*}$ & 85 & $17 / 19$ \\
\hline Ciprofloxacin & 7.02 & 281 & $215 / 156^{*}$ & 85 & $17 / 17$ \\
\hline Enrofloxacin & 5.50 & 265 & $172 / 156^{*}$ & 85 & $17 / 17$ \\
\hline Lomefloxacin & 0.94 & 215 & $108 / 156^{*}$ & 85 & $22 / 13$ \\
\hline Danofloxacin & 9.78 & 311 & $108 / 156^{*}$ & 85 & $29 / 19$ \\
\hline Orbifloxacin & 9.28 & 311 & $108 / 156^{*}$ & 85 & $29 / 19$ \\
\hline Difloxacin & 4.59 & 251 & $108 / 156^{*}$ & 85 & $25 / 16$ \\
\hline Sarafloxacin & 8.34 & 285 & $108 / 156^{*}$ & 85 & $24 / 15$ \\
\hline Sparfloxacin & 8.98 & 301 & $108 / 156^{*}$ & 85 & $25 / 17$ \\
\hline Oxolinic acid & 8.84 & 277.3 & $107.7 / 156^{*}$ & 80 & $20 / 12$ \\
\hline Flumequine & 8.90 & 285 & $108 / 156^{*}$ & 80 & $37 / 24$ \\
\hline Pefloxacin & 11.05 & 315 & $160 / 158^{*}$ & 80 & $23 / 25$ \\
\hline Nalidixic acid & 2.93 & 215 & $108 / 156^{*}$ & 80 & $20 / 10$ \\
\hline Sulfisoxazole & 9.15 & 268 & $112.8 / 155.8^{*}$ & 80 & $20 / 15$ \\
\hline Sulfisomidine & 9.90 & 329.3 & $208.1 / 313.1^{*}$ & 110 & $49 / 49$ \\
\hline Sulfathiazole & 11.84 & 331.3 & $239 / 316^{*}$ & 110 & $42 / 33$ \\
\hline Sulfapyridine & 10.55 & 321 & $257 / 152^{*}$ & -60 & $-16 /-26$ \\
\hline Sulfamonomethoxine & 10.29 & 356 & $185 / 336^{*}$ & -60 & $-14 /-27$ \\
\hline Sulfadoxine-D3 & 9.29 & 314 & 156 & 85 & 17 \\
\hline Sulfadimethoxine-D6 & 9.78 & 317 & 156 & 85 & 20 \\
\hline Norfloxacin-D5 & 7.36 & 325.3 & 307.2 & 80 & 30 \\
\hline Ciprofloxacin-D8 & 7.57 & 340.3 & 322.3 & 85 & 29 \\
\hline Enrofloxacin-D5 & 8.24 & 365.3 & 321.3 & 94 & 28 \\
\hline Malachite green-D5 & 9.92 & 334.3 & 318.2 & 120 & 52 \\
\hline Leucomalachite green-D6 & 11.79 & 337.3 & 322.3 & 110 & 31 \\
\hline Chloramphenicol-D5 & 10.54 & 326 & 157 & -60 & -26 \\
\hline
\end{tabular}

*Transitions for quantification.

columns (500 mg) and HLB cartridges (6 mL, $200 \mathrm{mg}$ ) were set up for cleaning up and enriching the aqueous solutions of sample extracts. SPE Cleanert Alumina N column was placed on top of the HLB cartridge for removing polar impurities. Five $\mathrm{mL}$ of sample extract was passed through the cartridge at a flow rate of $5 \mathrm{~mL} \cdot \mathrm{min}^{-1}$. Then, the SPE
Cleanert Alumina N column was removed, and the HLB cartridge was rinsed with $5 \mathrm{~mL}$ water to remove weakly bound impurities. The analytes were eluted with $8 \mathrm{~mL} 0.01 \%$ formic acid-methanol, and then the eluates were evaporated to dryness under a gentle stream of nitrogen at $35^{\circ} \mathrm{C}$. The dried extract was reconstituted in $2 \mathrm{~mL}$ of $10 \%$ acetonitrile 
TABLE 2: Instrument conditions for target compounds analysis.

\begin{tabular}{|c|c|c|c|c|c|c|c|c|c|}
\hline \multirow[t]{3}{*}{ Ionization mode } & & $\begin{array}{l}\mathrm{ESI}^{+} \\
\mathrm{ESI}^{-}\end{array}$ & & & & & & & \\
\hline & Mobile phase & $\begin{array}{l}\text { A } \\
\text { B }\end{array}$ & $\begin{array}{l}0.1 \% \text { formic acid and } 2 \mathrm{mM} \text { ammonium acetate } \\
0.1 \% \text { formic acid and acetonitrile }\end{array}$ & & & & & & \\
\hline & Gradient list & Time (min) & 0.1 & 8 & 10 & 11 & 13 & 13.11 & 19 \\
\hline \multirow[t]{6}{*}{ LC condition } & & A (\%) & 98 & 20 & 20 & 2 & 2 & 98 & 98 \\
\hline & & B (\%) & 2 & 80 & 80 & 98 & 98 & 2 & 2 \\
\hline & Total flow & $0.25 \mathrm{~mL} / \mathrm{min}$ & & & & & & & \\
\hline & Column temperature & $40^{\circ} \mathrm{C}$ & & & & & & & \\
\hline & Source temperature & $550^{\circ} \mathrm{C}$ & & & & & & & \\
\hline & Curtain gas & $20 \mathrm{psi}$ & & & & & & & \\
\hline \multirow{4}{*}{ MS condition } & Collision gas & Medium & & & & & & & \\
\hline & Gas1 & 60 psi & & & & & & & \\
\hline & Gas2 & $40 \mathrm{psi}$ & & & & & & & \\
\hline & Ion spray voltage & $5000 \mathrm{v}$ & & & & & & & \\
\hline
\end{tabular}

TABle 3: The ratio of substrong fragment area to quantitative ion area.

\begin{tabular}{lr}
\hline Ion ratio of analytes (\%) & Relative standard deviation (\%) \\
\hline$>50$ & \pm 20 \\
$20-50$ (not including 20) & \pm 25 \\
$10-20$ (not including 10) & \pm 30 \\
$\leq 10$ & \pm 50 \\
\hline
\end{tabular}

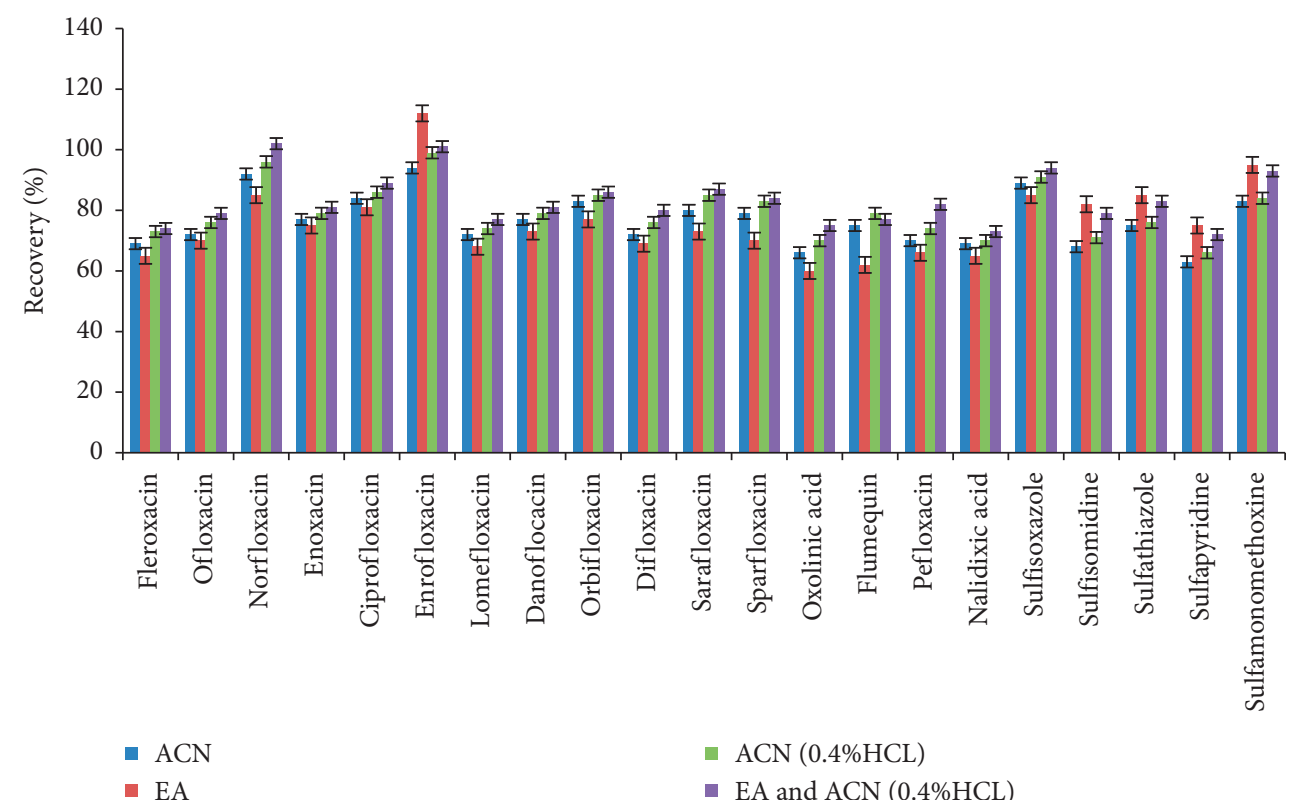

(a)

Figure 1: Continued. 


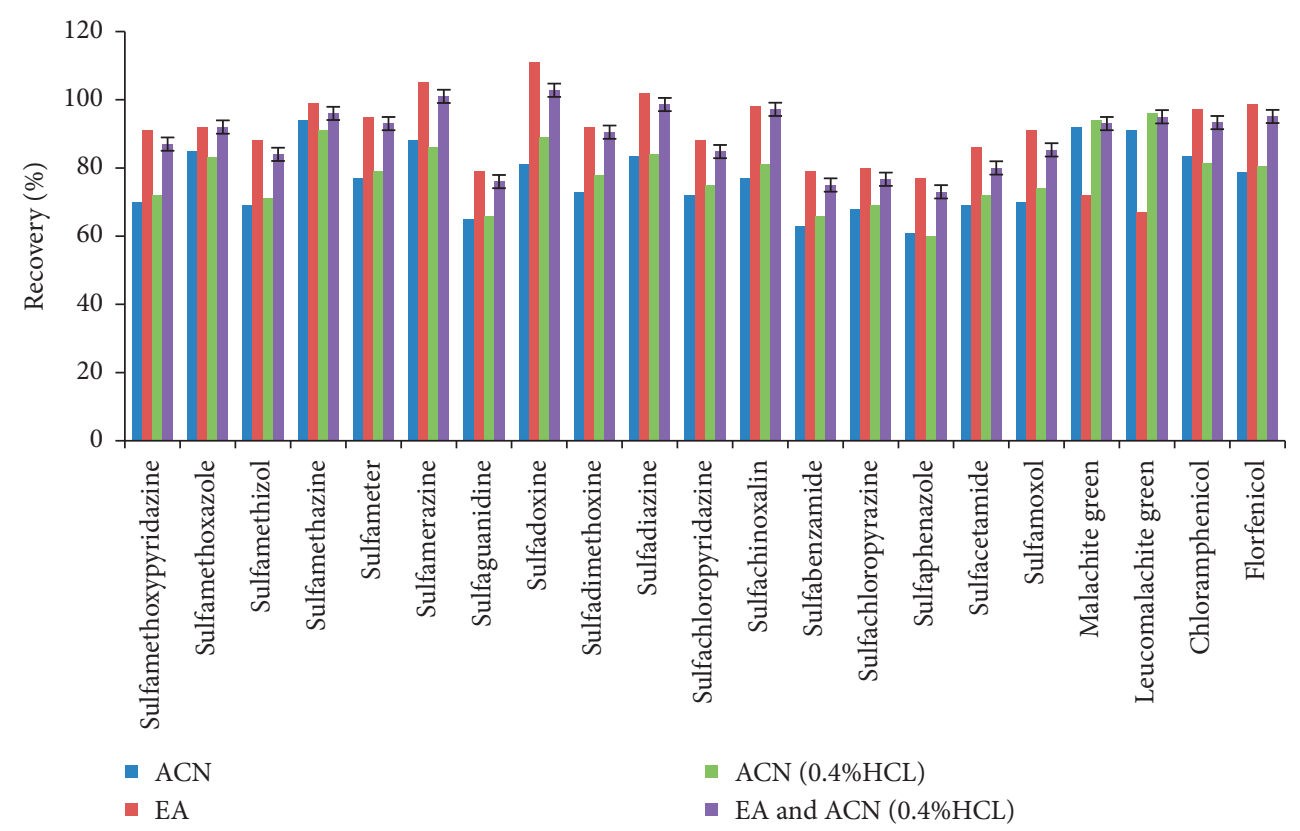

(b)

Figure 1: Effect of different 439 extraction solvents on recovery (\%) of 42 compounds.

solution. The reconstituted solution was mixed on a vortex mixer for $2 \mathrm{~min}$ and then $1 \mathrm{~mL}$ reconstituted solution was transferred to a $2 \mathrm{~mL}$ centrifuge tube and centrifuged (Eppendof 5424, Hamburg, Germany) at $12,638 \times g$ for $10 \mathrm{~min}$ at $4^{\circ} \mathrm{C}$. The supernatant was filtered through a $0.2 \mu \mathrm{m}$ syringe filter (GHP ACRODISC $130.2 \mu \mathrm{m}$ ) into a glass LC vial for analysis by LC-MS/MS.

\subsection{Instrumental Conditions. In order to achieve better} sensitivity and selectivity, the MS parameters were optimized by infusing a standard solution of $0.2 \mu \mathrm{g} \mathrm{mL}^{-1}$ of each analyte. Collision energies were optimized in order to find the most abundant product ions. The work monitored two fragments, selecting the most intensive transition for quantification and another for confirmation. The optimization of the ESI source temperature, curtain gas, ion spray voltage, Gas1, and Gas2 in positive and negative mode by flow injection analysis (FIA) has increased the method's sensitivity and the ionization's efficiency. The optimization of MS parameters carried out in the MS/MS and the optimization of the ESI in positive and negative mode are shown in Table 1.

The target compounds were analyzed by LC-MS/MS (SHIMADZU liquid chromatography LC-30AD system coupled to an AB 5500 Qtrap triple quadrupole mass spectrometer) in multiple-reaction monitoring (MRM) mode. The analyses were performed in the negative mode for two target compounds (CAP and FF) and in the positive mode for the other compounds. Nitrogen was used as the collision gas. Table 2 shows LC and MS parameters. Mass spectrometric conditions were optimized using an optimizer (AB, Palo Alto, CA, USA) for collision energy (CE) and declustering potential (DP). Table 2 shows MS/MS transitions for quantification and confirmation as well as CE and DP values optimized for each of the selected compounds.

Different kinds of LC columns were tested for separation of compounds. When a Thermo $\mathrm{C}_{18}$ column $(100 \times 2.1 \mathrm{~mm}$, $5 \mu \mathrm{m}$ ) was used for all the compounds with the mobile phase of A: $0.1 \%$ formic acid with $2 \mathrm{mM}$ ammonium acetate and B: $0.1 \%$ formic acid in acetonitrile, some compounds could not produce a conclusive peak shape and sensitivity and could not be separated by chromatography. By using a CAPCELL PAK $C_{18}$ MG II column $(100 \times 2.0 \mathrm{~mm}, 5 \mu \mathrm{m})$ for all the compounds with the mobile phase of $\mathrm{A}: 0.1 \%$ formic acid with $2 \mathrm{mM}$ ammonium acetate and B: $0.1 \%$ formic acid in acetonitrile, although some compounds could be separated by chromatography, other compounds could not produce a conclusive peak shape, whereas Shim-pack XR-ODS $(75 \mathrm{~mm} \times 2.1 \mathrm{~mm}, 2.2 \mu \mathrm{m})$ gave a narrow peak, better sensitivity, and calibration curve linearity for each analyte compared to the two other columns. Based on the comparative experiment, Shim-pack XR-ODS $(75 \mathrm{~mm} \times 2.1 \mathrm{~mm}$, $2.2 \mu \mathrm{m}$ ) was chosen for the best separation of all target compounds.

When 42 compounds are analyzed simultaneously and the chemical structures of the analytes differ greatly and the optimization of chromatographic separation is very difficult. There are fifteen analytes, oxolinic acid, olumequin, oulfaguanidine, sulfacetamide, sulfamethoxazole, sulfisoxazole, sulfamethazine, sulfisomidine, sulfameter, sulfamethoxypyridazine, sulfamonomethoxine, sulfachloropyridazine, sulfachloropyrazine, sulfadoxine, and sulfadimethoxine, that have to be separated by chromatography due to the same transitions using Shim-pack XR-ODS $(75 \mathrm{~mm} \times 2.1 \mathrm{~mm}$, $2.2 \mu \mathrm{m})$ LC column. During method optimization, the mobile phase compositions tested were (1) A: $0.1 \%$ formic 
TABLE 4: Linearity for the determination of analytes in fish by liquid chromatography-tandem mass spectrometry.

\begin{tabular}{|c|c|c|c|c|}
\hline Analyte & Standard equation & $R^{2}$ & Standard addition equation $^{\text {a }}$ & $R^{2}$ \\
\hline Fleroxacin & $y=0.022 x+0.006$ & 0.994 & $y=0.060 x+0.053$ & 0.991 \\
\hline Ofloxacin & $y=0.027 x+0.069$ & 0.999 & $y=0.075 x+0.011$ & 0.999 \\
\hline Norfloxacin & $y=0.014 x+0.003$ & 0.999 & $y=0.047 x+0.002$ & 0.998 \\
\hline Enoxacin & $y=0.077 x+0.349$ & 0.998 & $y=0.197 x+0.059$ & 1.000 \\
\hline Ciprofloxacin & $y=0.017 x+0.129$ & 0.994 & $y=0.034 x+0.078$ & 0.994 \\
\hline Enrofloxacin & $y=0.081 x+0.217$ & 0.996 & $y=0.030 x+0.038$ & 0.999 \\
\hline Lomefloxacin & $y=0.010 x+0.034$ & 0.991 & $y=0.015 x+0.062$ & 0.999 \\
\hline Danofloxacin & $y=0.024 x+0.037$ & 0.999 & $y=0.031 x+0.092$ & 0.999 \\
\hline Orbifloxacin & $y=0.016 x+0.033$ & 0.995 & $y=0.007 x+0.069$ & 0.998 \\
\hline Difloxacin & $y=0.012 x+0.021$ & 0.994 & $y=0.005 x+0.066$ & 0.997 \\
\hline Sarafloxacin & $y=0.007 x+0.015$ & 0.999 & $y=0.003 x+0.022$ & 0.999 \\
\hline Sparfloxacin & $y=0.015 x+0.035$ & 0.999 & $y=0.005 x+0.073$ & 0.993 \\
\hline Oxolinic acid & $y=0.899 x+3.76$ & 0.996 & $y=0.501 x+0.034$ & 0.992 \\
\hline Flumequine & $y=0.838 x+1.75$ & 0.998 & $y=0.359 x+0.022$ & 0.998 \\
\hline Pefloxacin & $y=0.218 x+0.344$ & 0.993 & $y=0.445 x+0.027$ & 0.999 \\
\hline Nalidixic acid & $y=1.18 x+6.58$ & 0.993 & $y=0.598 x+0.067$ & 0.995 \\
\hline Sulfisoxazole & $y=0.014 x+0.048$ & 0.992 & $y=0.090 x+0.056$ & 0.993 \\
\hline Sulfisomidine & $y=0.009 x+0.054$ & 0.993 & $y=0.007 x+0.091$ & 0.992 \\
\hline Sulfathiazole & $y=0.023 x+0.075$ & 0.995 & $y=0.019 x+0.109$ & 0.996 \\
\hline Sulfapyridine & $y=0.018 x+0.005$ & 0.995 & $y=0.063 x+0.322$ & 0.999 \\
\hline Sulfamonomethoxine & $y=0.090 x+0.022$ & 0.997 & $y=0.082 x+0.181$ & 0.998 \\
\hline Fleroxacin & $y=0.030 x+0.023$ & 0.991 & $y=0.022 x+0.306$ & 0.994 \\
\hline Ofloxacin & $y=0.024 x+0.010$ & 0.992 & $y=0.013 x+0.032$ & 0.999 \\
\hline Norfloxacin & $y=0.021 x+0.004$ & 0.998 & $y=0.022 x+0.231$ & 1.000 \\
\hline Enoxacin & $y=0.009 x+0.014$ & 0.997 & $y=0.006 x+0.123$ & 0.996 \\
\hline Ciprofloxacin & $y=0.009 x+0.065$ & 0.996 & $y=0.007 \times 0.118$ & 1.000 \\
\hline Enrofloxacin & $y=0.014 x+0.050$ & 0.998 & $y=0.012 x+0.124$ & 0.992 \\
\hline Lomefloxacin & $y=0.003 x+0.015$ & 0.999 & $y=0.002 x+0.038$ & 0.999 \\
\hline Danofloxacin & $y=0.046 x+0.099$ & 0.993 & $y=0.015 x+0.018$ & 0.998 \\
\hline Orbifloxacin & $y=0.053 x+0.046$ & 0.999 & $y=0.039 x+0.097$ & 0.999 \\
\hline Difloxacin & $y=0.011 x+0.052$ & 1.000 & $y=0.009 x+0.176$ & 0.992 \\
\hline Sarafloxacin & $y=0.033 x+0.133$ & 0.998 & $y=0.018 x+0.083$ & 0.997 \\
\hline Sparfloxacin & $y=0.006 x+0.043$ & 0.999 & $y=0.002 x+0.098$ & 0.994 \\
\hline Oxolinic acid & $y=0.033 x+0.094$ & 0.9988 & $y=0.012 x+0.054$ & 0.997 \\
\hline Flumequine & $y=0.002 x+0.001$ & 0.999 & $y=0.001 x+0.014$ & 0.993 \\
\hline Pefloxacin & $y=0.017 x+0.033$ & 1.000 & $y=0.007 x+0.022$ & 0.992 \\
\hline Nalidixic acid & $y=0.004 x+0.001$ & 0.999 & $y=0.004 x+0.028$ & 0.993 \\
\hline Sulfisoxazole & $y=0.009 x+0.018$ & 0.998 & $y=0.004 x+0.127$ & 0.993 \\
\hline Sulfisomidine & $y=0.614 x+0.085$ & 0.995 & $y=0.212 x+0.224$ & 0.999 \\
\hline Sulfathiazole & $y=0.558 x+0.001$ & 0.998 & $y=0.191 x+0.104$ & 0.997 \\
\hline Sulfapyridine & $y=0.338 x+0.024$ & 0.999 & $y=0.087+0.012$ & 0.990 \\
\hline Sulfamonomethoxine & $y=0.324 x+0.012$ & 1.000 & $y=0.092 x+0.022$ & 0.992 \\
\hline
\end{tabular}

acid in water, B: $0.1 \%$ formic acid in methanol, (2) A: $0.1 \%$ formic acid with $2 \mathrm{mM}$ ammonium acetate, B: $0.1 \%$ formic acid in methanol, (3) A: $0.1 \%$ formic acid with $5 \mathrm{mM}$ ammonium acetate, B: $0.1 \%$ formic acid in methanol, (4) A: $0.1 \%$ formic acid in water, B: $0.1 \%$ formic acid in acetonitrile, (5) A: $0.1 \%$ formic acid with $2 \mathrm{mM}$ ammonium acetate, $\mathrm{B}$ : $0.1 \%$ formic acid in acetonitrile, and (6) A: $0.1 \%$ formic acid with $5 \mathrm{mM}$ ammonium acetate, B: $0.1 \%$ formic acid in acetonitrile. The mobile phase with A: $0.1 \%$ formic acid with $2 \mathrm{mM}$ ammonium acetate and B: $0.1 \%$ formic acid in acetonitrile can achieve a better chromatographic resolution and peak sensitivity for all compounds compared to using other mobile phases.

\subsection{Method Validation}

2.4.1. Linearity. The linearity was evaluated by standard addition calibration curves at different spiked levels. Calibration curves were obtained for Grass Carp matrix with concentrations based on the response of each analyte. The compounds were categorized into three groups: group 1 included sulfisomidine, sulfathiazole, sulfapyridine, sulfamonomethoxine, sulfamethoxypyridazine, sulfamethoxazole, sulfamethizole, sulfamethazine, sulfameter, sulfaguanidine, sulfadoxine, sulfadimethoxine, sulfadiazine, sulfachloropyridazine, sulfachinoxalin, sulfabenzamide, sulfaphenazole, sulfacetamide, sulfamoxol, fleroxacin, ofloxacin, enoxacin, enrofloxacin, danofloxacin, 
TABle 5: Parameters of UPLC-MS/MS for the 42 compounds in fish sample.

\begin{tabular}{|c|c|c|c|c|c|c|c|}
\hline Analyte & $\begin{array}{l}\text { Spiked } \\
\text { levels }\end{array}$ & $\begin{array}{c}\text { Recoveries } R \\
(\%)\end{array}$ & Internal standard & $\begin{array}{c}\text { LOD } \\
\mu \mathrm{g} \cdot \mathrm{kg}^{-1}\end{array}$ & $\begin{array}{c}\mathrm{LOQ} \\
\mu \mathrm{g} \cdot \mathrm{kg}^{-1}\end{array}$ & $\begin{array}{c}\text { Intraday RSD(\%) } \\
\mu \mathrm{g} \cdot \mathrm{kg}^{-1}\end{array}$ & $\begin{array}{c}\text { Inter-day } \\
\text { RSD } \\
(\%) \mu \mathrm{g} \cdot \mathrm{kg}^{-1} \\
\end{array}$ \\
\hline Fleroxacin & $0.5 / 1 / 4$ & $71.2 / 73.4 / 75.5$ & Enrofloxacin-D5 & 0.1 & 0.4 & 3.6 & 4.1 \\
\hline Sulfamethoxazole & $0.5 / 1 / 4$ & $83.6 / 81.5 / 92.4$ & Sulfadimethoxine-D6 & 0.1 & 0.4 & 1.4 & 2.2 \\
\hline Ofloxacin & $0.5 / 1 / 4$ & $77.6 / 74.8 / 81.2$ & Enrofloxacin-D5 & 0.1 & 0.4 & 1.2 & 1.1 \\
\hline Sulfamethizol & $0.5 / 1 / 4$ & $74.4 / 78.8 / 82.5$ & Sulfadimethoxine-D6 & 0.1 & 0.4 & 2.7 & 3.3 \\
\hline Norfloxacin & $1 / 2 / 10$ & 101/102/106 & Norfloxacin-D5 & 0.25 & 1.0 & 4.4 & 3.8 \\
\hline Sulfamethazine & $0.5 / 1 / 4$ & $92.2 / 95.4 / 96.7$ & Sulfadimethoxine-D6 & 0.1 & 0.4 & 1.5 & 1.7 \\
\hline Enoxacin & $0.5 / 1 / 4$ & $75.5 / 73.6 / 78.9$ & Enrofloxacin-D5 & 0.1 & 0.4 & 1.8 & 2.3 \\
\hline Sulfameter & $0.5 / 1 / 4$ & $88.1 / 90.3 / 94.6$ & Sulfadimethoxine-D6 & 0.1 & 0.4 & 1.8 & 2.5 \\
\hline Ciprofloxacin & $1 / 2 / 10$ & $82.1 / 85.4 / 88.6$ & Ciprofloxacin-D8 & 0.25 & 1.0 & 2.4 & 2.6 \\
\hline Sulfamerazine & $0.5 / 1 / 4$ & $95.5 / 94.6 / 1003$ & Sulfadimethoxine-D6 & 0.1 & 0.4 & 1.3 & 3.4 \\
\hline Enrofloxacin & $0.5 / 1 / 4$ & 101/104/107 & Enrofloxacin-D5 & 0.1 & 0.4 & 2.7 & 1.2 \\
\hline Sulfaguanidine & $0.5 / 1 / 4$ & $71.6 / 72.4 / 76$ & Sulfadimethoxine-D6 & 0.1 & 0.4 & 1.3 & 2.3 \\
\hline Lomefloxacin & $1 / 2 / 10$ & $75.5 / 75 / 77.3$ & Enrofloxacin-D5 & 0.25 & 1.0 & 3.4 & 3.7 \\
\hline Sulfadoxine & $0.5 / 1 / 4$ & $105 / 107 / 104$ & Sulfadoxine-D3 & 0.1 & 0.4 & 2.1 & 4.3 \\
\hline Danofloxacin & $0.5 / 1 / 4$ & $76.6 / 76.7 / 81$ & Enrofloxacin-D5 & 0.1 & 0.4 & 1.6 & 1.9 \\
\hline Sulfadimethoxine & $0.5 / 1 / 4$ & 83.2/87.2/91.8 & Sulfadimethoxine-D6 & 0.1 & 0.4 & 1.4 & 2.7 \\
\hline Orbifloxacin & $0.5 / 1 / 4$ & 83.6/83.8/87 & Enrofloxacin-D5 & 0.1 & 0.4 & 1.1 & 1.8 \\
\hline Sulfadiazine & $0.5 / 1 / 4$ & $92.6 / 93.2 / 103$ & Sulfadoxine-D3 & 0.1 & 0.4 & 2.3 & 3.8 \\
\hline Difloxacin & $0.5 / 1 / 4$ & $72.2 / 77.5 / 77$ & Enrofloxacin-D5 & 0.1 & 0.4 & 1.5 & 1.4 \\
\hline Sulfachloropyridazine & $0.5 / 1 / 4$ & $82.6 / 82.7 / 84.2$ & Sulfadimethoxine-D6 & 0.1 & 0.4 & 2.2 & 4.5 \\
\hline Sarafloxacin & $1 / 2 / 10$ & $84.9 / 77.5 / 83.4$ & Sulfadimethoxine-D6 & 0.25 & 1.0 & 1.4 & 2.2 \\
\hline Sulfachinoxalin & $0.5 / 1 / 4$ & $67.2 / 71 / 71.5$ & Sulfadimethoxine-D6 & 0.1 & 0.4 & 2.6 & 4.1 \\
\hline Sparfloxacin & $1 / 2 / 10$ & $84.4 / 86.5 / 78.3$ & Enrofloxacin-D5 & 0.25 & 1.0 & 1.8 & 2.5 \\
\hline Sulfabenzamide & $0.5 / 1 / 4$ & $71.6 / 72.4 / 75.8$ & Sulfadimethoxine-D6 & 0.1 & 0.4 & 3.1 & 5.6 \\
\hline Oxolinic acid & $0.5 / 1 / 4$ & $69.2 / 71.4 / 73.3$ & Enrofloxacin-D5 & 0.1 & 0.4 & 2.7 & 3.2 \\
\hline Sulfachloropyrazine & $2001 / 2 / 10$ & 73.7/81/76.3 & Sulfadimethoxine-D6 & 0.25 & 1.0 & 2.4 & 3.5 \\
\hline Flumequine & $0.5 / 1 / 4$ & $68.2 / 72.2 / 77.5$ & Enrofloxacin-D5 & 0.1 & 0.4 & 1.7 & 1.6 \\
\hline Sulfaphenazole & $0.5 / 1 / 4$ & $70.4 / 72.3 / 73.8$ & Sulfadimethoxine-D6 & 0.1 & 0.4 & 3.5 & 2.8 \\
\hline Pefloxacin & $0.5 / 1 / 4$ & $80.2 / 79 / 82.4$ & Enrofloxacin-D5 & 0.1 & 0.4 & 3.5 & 3.8 \\
\hline Sulfacetamide & $0.5 / 1 / 4$ & $74.2 / 75 / 80$ & Sulfadimethoxine-D6 & 0.1 & 0.4 & 3.7 & 3.5 \\
\hline Nalidixic acid & $0.5 / 1 / 4$ & $69.6 / 69.8 / 73.5$ & Enrofloxacin-D5 & 0.1 & 0.4 & 5.2 & 6.7 \\
\hline Sulfamoxol & $0.5 / 1 / 4$ & 83.6/85.2/87.8 & Sulfadimethoxine-D6 & 0.1 & 0.4 & 3.3 & 4.6 \\
\hline Sulfisoxazole & $1 / 2 / 10$ & 93.5/93/89.8 & Sulfadimethoxine-D6 & 0.25 & 1.0 & 1.1 & 2.4 \\
\hline Sulfamethoxypyridazine & $0.5 / 1 / 4$ & $78 / 81.9 / 86.2$ & Sulfadimethoxine-D6 & 0.1 & 0.4 & 1.7 & 1.8 \\
\hline Sulfisomidine & $1 / 2 / 10$ & $73.4 / 77 / 82.2$ & Sulfadimethoxine-D6 & 0.25 & 1.0 & 2.5 & 2.2 \\
\hline Sulfathiazole & $0.5 / 1 / 4$ & $83.8 / 80.7 / 81.5$ & Sulfadimethoxine-D6 & 0.1 & 0.4 & 2.6 & 3.3 \\
\hline Sulfapyridine & $0.5 / 1 / 4$ & $67 / 69.8 / 72.5$ & Sulfadimethoxine-D6 & 0.1 & 0.4 & 4.4 & 7.1 \\
\hline Sulfamonomethoxine & $0.5 / 1 / 4$ & $87 / 89.4 / 92$ & Sulfadimethoxine-D6 & 0.1 & 0.4 & 1.2 & 1.9 \\
\hline Malachite green & $0.5 / 1 / 4$ & $91 / 93.3 / 92.2$ & Malachite green-D5 & 0.1 & 0.4 & 2.6 & 1.6 \\
\hline Leucomalachite green & $0.5 / 1 / 4$ & $86.5 / 91.9 / 92.8$ & $\begin{array}{c}\text { Leucomalachite green- } \\
\text { D6 }\end{array}$ & 0.1 & 0.4 & 1.9 & 4.2 \\
\hline Chloramphenicol & $0.8 / 4.0 / 20$ & $91.6 / 95.2 / 106$ & Chloramphenicol-D5 & 0.15 & 0.5 & 1.6 & 3.8 \\
\hline Florfenicol & $0.8 / 4.0 / 20$ & 87.9/92.3/98 & Chloramphenicol-D5 & 0.15 & 0.5 & 2.1 & 1.7 \\
\hline
\end{tabular}

orbifloxacin, difloxacin, oxolinic acid, flumequine, pefloxacin, nalidixic acid, leucomalachite green, and malachite green, with the following spiking levels: $0.4,1,2,5,10,20$, and $50 \mu \mathrm{g} \mathrm{kg}^{-1}$. Group 2 included sulfisoxazole, sulfamerazine, sulfachloropyrazine, norfloxacin, ciprofloxacin, lomefloxacin, sarafloxacin, and sparfloxacin, with the following spiking levels: $1,2,5,10,20,50$, and $100 \mu \mathrm{g} \mathrm{kg}^{-1}$. Group 3 included chloramphenicol and florfenicol with the following spiking levels: $0.5,1.0,2,5,10$, and $20 \mu \mathrm{g} \mathrm{kg}^{-1}$.

2.4.2. Accuracy and Precision. The recovery was used to evaluate the accuracy of the method. Six replicates of spiked samples at three concentration levels were prepared. The concentrations of spiked samples were calculated by calibration curves. The recovery was determined by means of the measured concentration. The precision, expressed as relative standard deviation (RSD), was determined by intra- and interday assays.

2.4.3. Limit of Detection and Limit of Quantification. The LOD and LOQ were determined at a signal-to-noise ratio $(\mathrm{S} / \mathrm{N})$ of about 3 and 10 , respectively.

(1) Qualitative Analysis. Positive identification of compounds in fish samples was based on the criteria for retention time (deviation within 5\%). The ion ratio deviation of analytes and the standard should accord with Table 3 . The 


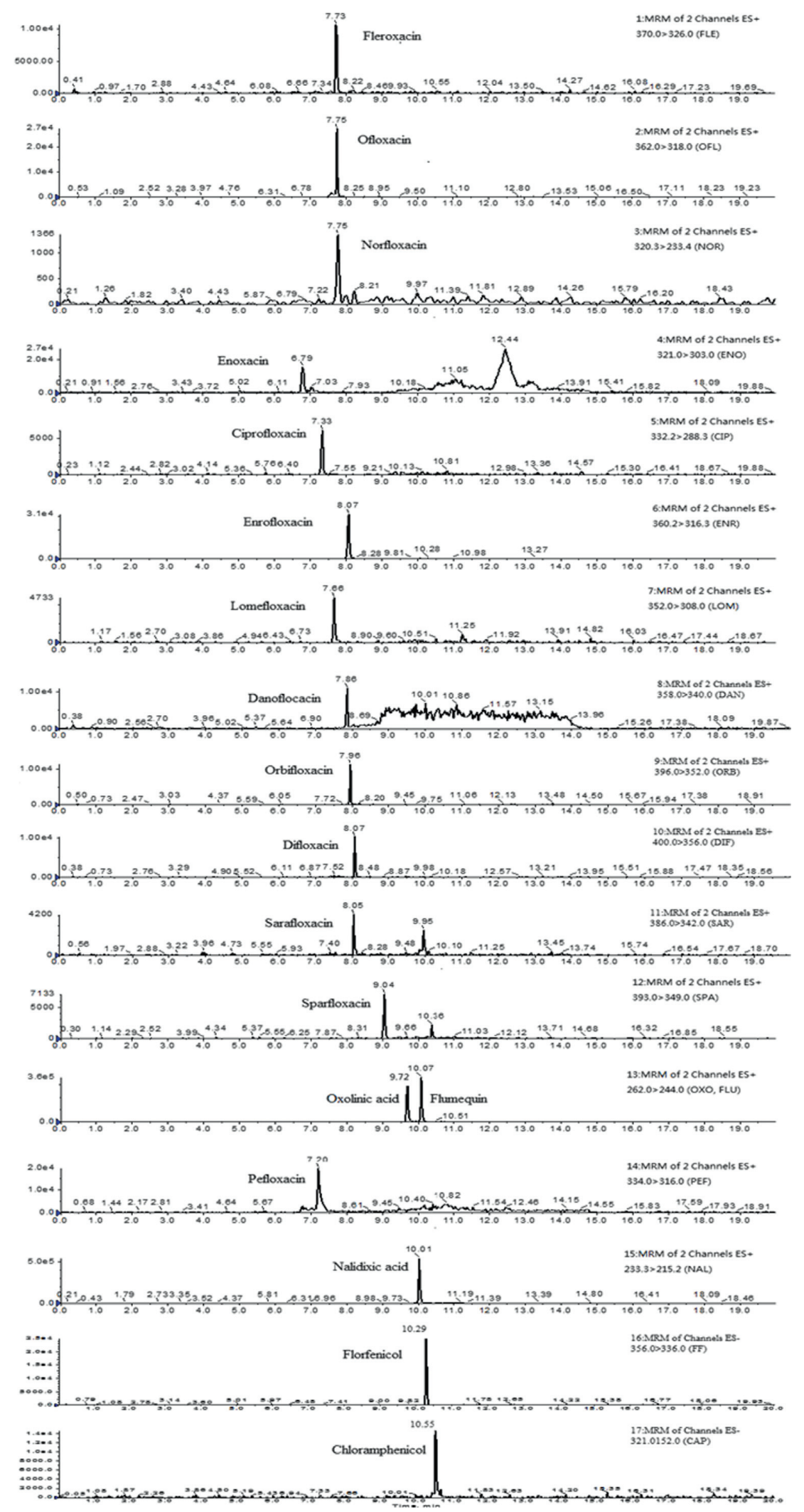

(a)

FIGURE 2: Continued. 


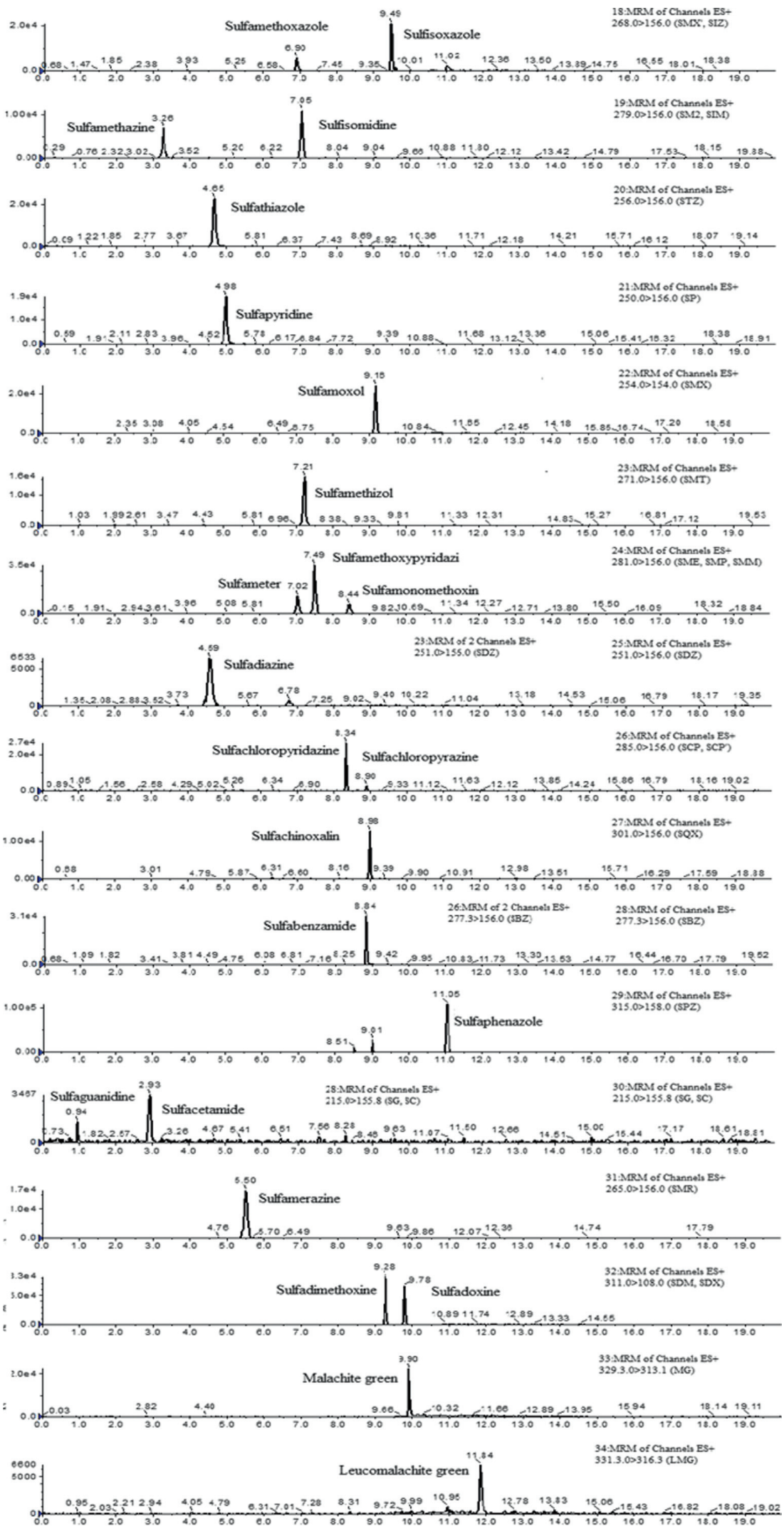

(b)

FIgURE 2: UPLC-MS/MS of 455 chromatograms of spiked fish muscle sample at LOQ level. 
TABLE 6: Confirmatory and quantitative analysis of samples.

\begin{tabular}{|c|c|c|c|c|}
\hline Analytes & Tissue & Residue amount $\left(\mu \mathrm{g} \cdot \mathrm{kg}^{-1}\right)$ & Ratio of analyte in sample (\%) & Ratio of analyte in standard (\%) \\
\hline \multirow{3}{*}{ Sulfamethazine } & Fish sample-1 & 41.3 & 30.2 & \multirow{3}{*}{37.1} \\
\hline & Fish sample-2 & 31.5 & 33.4 & \\
\hline & Fish sample-3 & 104 & 35.1 & \\
\hline \multirow{6}{*}{ Enrofloxacin } & Fish sample- 4 & 65.1 & 42.2 & \multirow{6}{*}{48.8} \\
\hline & Fish sample-5 & 14.8 & 40.3 & \\
\hline & Fish sample-6 & 15.5 & 44.6 & \\
\hline & Fish sample-7 & 73.6 & 51.5 & \\
\hline & Fish sample- 8 & 45.5 & 57.7 & \\
\hline & Fish sample-9 & 112 & 53.6 & \\
\hline \multirow{2}{*}{ Ciprofloxacin } & Fish sample-4 & 14.6 & 52.2 & \multirow{2}{*}{53.7} \\
\hline & Fish sample-7 & 16.3 & 55.6 & \\
\hline
\end{tabular}

method was satisfactory in terms of linearity, recovery, precision, and analytical limits under the requirements of the US FDA criteria.

\section{Results and Discussion}

3.1. Optimization of Extraction for Samples. In order to perform the simultaneous detection of SAs, QNs, chloramphenicol, florfenicol, leucomalachite green, and malachite green, the extraction method must be capable of effectively extracting each compound from the complex fish sample matrix. The extraction procedure is a critical step because it must be able to perform a good recovery of several compounds with different chemical properties. To optimize the extraction procedure, four different solvents, acetonitrile, ethyl acetate, $0.4 \%$ hydrochloric acid in acetonitrile, and ethyl acetate followed by $0.4 \%$ hydrochloric acid in acetonitrile, were evaluated as extraction solvents. Figure 1 shows that an acceptable recovery of compounds was only obtained by the two-step extraction utilizing ethyl acetate followed by $0.4 \%$ hydrochloric acid in acetonitrile. The use of acetonitrile and acidified acetonitrile as extraction solvents provided a better recovery of QNs, leucomalachite green, and malachite green, compared to SAs. However, the extraction efficiency of acidified acetonitrile was higher than that of acetonitrile. Using ethyl acetate as extraction solvents provided a better recovery of SAs, chloramphenicol, and florfenicol than QNs, leucomalachite green, and malachite green. The test results showed that acidified acetonitrile and ethyl acetate are suitable for extraction of all compounds because it provided the most satisfactory recoveries from the spiked samples.

Different kinds of extraction methods using a vortex mixer, ultrasonic bath, and homogenizer were tested for this experiment. In this work, extraction efficiency using the vortex was low. The ultrasonic bath yielded high extraction efficiency, which may be related to the state of the pulverized matrix, but poor stability. The more homogeneous and dispersed the matrix, the higher the extraction efficiency. The homogenizer provided higher stability, but poor extraction efficiency. Based on the above comparison, homogenization followed by ultrasonic extraction was selected as the optimized extraction procedure. Further optimization of ultrasonic treatment time was carried out by sonicating for $5,10,15,20,25,30$, and 35 minutes with an ultrasonic bath. The experimental results showed that recovery increased with longer sonication time; the recovery reached a peak at 25 minutes and tended to be stable. Ultrasonic treatment for 25 minutes was chosen. Five different kinds of microfiltration membranes SHIMADZU-GL II MCE $0.22 \mu \mathrm{m}$, GHP ACRODISC $130.2 \mu \mathrm{m}$, nylon $0.22 \mu \mathrm{m}$, AGILENT PTFE $0.2 \mu \mathrm{m}$, and Waters ACRODIC Syringe $0.2 \mu \mathrm{m}$ GHP were tested. When the sample extract went through a $0.22 \mu \mathrm{m}$ nylon membrane, the constituents in the microfiltration membrane were dissolved, which caused interference to QNs. SHIMADZU-GL II MCE $0.22 \mu \mathrm{m}$, AGILENT PTFE $0.2 \mu \mathrm{m}$, and Waters ACRODIC Syringe $0.2 \mu \mathrm{m}$ could not remove fat and protein successfully. Only the GHP ACRODISC $130.2 \mu \mathrm{m}$ membrane was suitable for filtration.

\subsection{Method Validation}

3.2.1. Linearity. Standard calibration curves were performed to achieve good accuracy and to compensate for the matrix effect and loss in the sample preparation. The calibration curves for analytes were constructed by plotting the ratio of analyte peak area to internal standard peak area in response of compounds $(y)$ versus concentration $(x)$ of each analyte which was expressed by the equation. The calibration curves were generated daily from the peak area responses of standards with concentrations ranging from 5 to $150 \mathrm{ng} \mathrm{mL} \mathrm{m}^{-1}$. Good linearity was found in the studied ranges with coefficients of determination $\left(R^{2} \geq 0.99\right)$.

3.2.2. Limit of Detection (LOD) and Limit of Quantification (LOQ). The negative samples were selected and spiked with the standard solution and then treated and analyzed following the method described above. The LODs and LOQs for each analyte were obtained from a signal-to-noise ratio (S/N) of about 3 and 10 in Table 4 . The LODs for norfloxacin, ciprofloxacin, lomefloxacin, sarafloxacin, sparfloxacin, sulfachloropyrazine, sulfisoxazole, and sulfisomidine were $0.25 \mu \mathrm{g} \mathrm{kg}^{-1}$ and the LOQs were $1.0 \mu \mathrm{g} \mathrm{kg}^{-1}$. The LODs for CAP and FF were $0.15 \mu \mathrm{g} \cdot \mathrm{kg}^{-1}$ and the LOQs were $0.5 \mu \mathrm{g} \mathrm{kg}^{-1}$. The LODs for other analytes were $0.1 \mu \mathrm{g} \mathrm{kg}^{-1}$ and the LOQs were $0.4 \mu \mathrm{g} \mathrm{kg}^{-1}$. 
3.2.3. Accuracy and Precision. All recoveries are between $70 \%$ and $120 \%$, except for oxolinic acid, flumequine, sulfapyridine, nalidixic acid, and sulfachinoxalin which show recoveries lower than $70 \%$ at some spiked levels, but the overall recovery is still acceptable. The recoveries shown in Table 5 are between $67.7 \%$ and $112.8 \%$. Precision was expressed as relative standard deviation (RSD). The results of intraday and interday and for all analytes are listed in Table 5. The precision of the intraday RSDs was less than $6.3 \%$ and the precision of the interday RSDs was less than $8.5 \%$ in the fish samples. Typical MRM chromatograms of spiked samples are shown in Figure 2.

3.3. Analysis of Real Samples. In this work, the method applicability was checked by analyzing 30 fish samples collected from local markets. The samples originated from Heilongjiang province and Beijing city and were collected in May 2017 according to the procedures required by the surveillance program of the Chinese Ministry of Agriculture. The most prevalent compounds were sulfamethazine and enrofloxacin, and two samples surpassed the established MRL of $100 \mu \mathrm{g} \mathrm{kg}^{-1}$ with values of $104 \mu \mathrm{g} \mathrm{kg}^{-1}$ and $112 \mu \mathrm{g} \mathrm{kg}^{-1}$ in fish samples. Ciprofloxacin was detected in almost all samples with high residual enrofloxacin samples because ciprofloxacin was a metabolite of enrofloxacin. The detected compounds were identified by means of retention time and ion ratio. Table 6 summarizes the confirmatory and the quantitative analysis of the nine fish samples.

\section{Conclusions}

A selective and rapid UHPLC-MS/MS confirmatory method was developed and validated for the simultaneous detection of 22 SAs and 16 QNs, chloramphenicol, florfenicol, malachite green, and leucomalachite green in fish. The present work was to optimize sample extraction, purification, and chromatographic separation. The method enables the determination of the analyte residues in the low range and is qualified to carry out risk warnings for drug residues in fish.

\section{Data Availability}

The data used to support the findings of this study are available from the corresponding author upon request.

\section{Disclosure}

Yongping Chen and Sudong Xia contributed equally to this work and should be considered co-first authors.

\section{Conflicts of Interest}

The authors declare that they have no conflicts of interest.

\section{Acknowledgments}

This research was supported by the National Key R\&D Program of China (2018YFD0900400) and Projects of Science and Technology of Tianjin (17YFNZNC00110).

\section{References}

[1] R. Kafaei, F. Papari, M. Seyedabadi et al., "Occurrence, distribution, and potential sources of antibiotics pollution in the water-sediment of the northern coastline of the Persian gulf, Iran," Science of the Total Environment, vol. 627, pp. 703-712, 2018.

[2] R. Zhang, J. Pei, R. Zhang et al., "Occurrence and distribution of antibiotics in mariculture farms, estuaries and the coast of the Beibu gulf, China: bioconcentration and diet safety of seafood," Ecotoxicology and Environmental Safety, vol. 154, pp. 27-35, 2018.

[3] L. J. Zhou, G. G. Ying, S. Liu et al., "Simultaneous determination of human and veterinary antibiotics in various environmental matrices by rapid resolution liquid chromatography-electrospray ionization tandem mass spectrometry," Journal of Chromatography A, vol. 1244, pp. 123-138, 2016.

[4] A. C. Johnson, M. D. Jürgens, R. J. Williams, K. Kümmerer, A. Kortenkamp, and J. P. Sumpter, "Do cytotoxic chemotherapy drugs discharged into rivers pose a risk to the environment and human health? An overview and UK case study," Journal of Hydrology, vol. 348, no. 1-2, pp. 167-175, 2008.

[5] R. M. Briones, A. K. Sarmah, and L. P. Padhye, "A global perspective on the use, occurrence, fate and effects of antidiabetic drug metformin in natural and engineered ecosystems," Environmental Pollution, vol. 219, pp. 1007-1020, 2016.

[6] D. J. Lapworth, N. Baran, M. E. Stuart, and R. S. Ward, "Emerging organic contaminants in groundwater: a review of sources, fate and occurrence," Environmental Pollution, vol. 163, pp. 287-303, 2012.

[7] S. Srivastava, R. Sinha, and D. Roy, "Toxicological effects of malachite green," Aquatic Toxicology, vol. 66, no. 3, pp. 319-329, 2004.

[8] E. L. E. Jester, J. I. Loader, H. A. F. Quintana, K. R. E. Said, R. A. Benner, and A. Abraham, "Analysis of chloramphenicol and two metabolites in crab and shrimp following waterborne exposure," Journal of Food Protection, vol. 81, no. 4, pp. 677-683, 2018.

[9] M. I. Chughtai, U. Maqbool, M. Iqbal, M. S. Shah, and T. Fodey, "Development of in-house ELISA for detection of chloramphenicol in bovine milk with subsequent confirmatory analysis by LC-MS/MS," Journal of Environmental Science and Health, Part B, vol. 52, no. 12, pp. 871-879, 2017.

[10] W. Tian, L. Gao, Y. Zhao, W. Peng, and Z. Chen, "Simultaneous determination of metronidazole, chloramphenicol and 10 sulfonamide residues in honey by LC-MS/MS," $A n$ alytical Methods, vol. 5, no. 5, pp. 1283-1288, 2013.

[11] F. Akrami-Mohajeri, Z. Derakhshan, M. Ferrante et al., "The prevalence and antimicrobial resistance of Listeria spp in raw milk and traditional dairy products delivered in Yazd, central Iran (2016)," Food and Chemical Toxicology, vol. 114, pp. 141-144, 2018.

[12] K.-X. Xu, M.-H. Guo, Y.-P. Huang, X.-D. Li, and J.-J. Sun, "Rapid and sensitive detection of malachite green in aquaculture water by electrochemical preconcentration and 
surface-enhanced Raman scattering," Talanta, vol. 180, pp. 383-388, 2018.

[13] M. Razi-Asrami, J. B. Ghasemi, N. Amiri, and S. J. Sadeghi, "Simultaneous spectrophotometric determination of crystal violet and malachite green in water samples using partial least squares regression and central composite design after preconcentration by dispersive solid-phase extraction," Environmental Monitoring and Assessment, vol. 189, no. 4, p. 196, 2017.

[14] D. Zhu, Q. Li, X. Pang, Y. Liu, X. Wang, and G. Chen, “A sensitive electrochemical impedance immunosensor for determination of malachite green and leucomalachite green in the aqueous environment," Analytical and Bioanalytical Chemistry, vol. 408, no. 20, pp. 593-600, 2016.

[15] S. K. Saxena, R. Rangasamy, A. A. Krishnan et al., "Simultaneous determination of multi-residue and multi-class antibiotics in aquaculture shrimps by UPLC-MS/MS," Food Chemistry, vol. 260, pp. 336-343, 2018.

[16] J. Xie, T. Peng, D.-D. Chen et al., "Determination of malachite green, crystal violet and their leuco-metabolites in fish by HPLC-VIS detection after immunoaffinity column clean-up," Journal of Chromatography B, vol. 913-914, pp. 123-128, 2014.

[17] A. A. Fallah and A. Barani, "Determination of malachite green residues in farmed rainbow trout in Iran," Food Control, vol. 40, no. 1, pp. 100-105, 2013.

[18] A. S. Tsagkaris, J. L. D. Nelis, G. M. S. Ross et al., "Critical assessment of recent trends related to screening andconfirmatory analytical methods for selected food contaminants and allergens," Trends in Analytical Chemistry, vol. 121, pp. 1-14, 2019.

[19] Z.-Z. Lin, H.-Y. Zhang, A.-h. Peng, Y.-D. Lin, L. Li, and Z.-Y. Huang, "Determination of malachite green in aquatic products based on magnetic molecularly imprinted polymers," Food Chemistry, vol. 200, pp. 32-37, 2016.

[20] R. Yamada, M. Kozono, T. Ohmori, F. Morimatsu, and M. Kitayama, "Simultaneous determination of residual veterinary drugs in bovine, porcine, and chicken muscle using liquid chromatography coupled with electrospray ionization tandem mass spectrometry," Bioscience, Biotechnology, and Biochemistry, vol. 70, no. 1, pp. 54-65, 2006.

[21] A. Freitas, J. Barbosa, and F. Ramos, "Multidetection of antibiotics in liver tissue by ultra-high-pressure-liquid-chromatography-tandem mass spectrometry," Journal of Chromatography B, vol. 976-977, pp. 49-54, 2015.

[22] W. Ben, Z. Qiang, C. Adams, H. Zhang, and L. Chen, "Simultaneous determination of sulfonamides, tetracyclines and tiamulin in swine wastewater by solid-phase extraction and liquid chromatography-mass spectrometry," Journal of Chromatography A, vol. 1202, no. 2, pp. 173-180, 2008.

[23] G. C. Bedendo, I. C. S. F. Jardim, and E. Carasek, "A simple hollow fiber renewal liquid membrane extraction method for analysis of sulfonamides in honey samples with determination by liquid chromatography-tandem mass spectrometry," Journal of Chromatography A, vol. 1217, no. 42, pp. 6449$6454,2010$.

[24] A. Economou, O. Petraki, D. Tsipi, and E. Botitsi, "Determination of a liquid chromatography-tandem mass spectrometry method for the determination of sulfonamides, trimethoprim and dapsone in honey and validation according to Commission Decision 2002/657/EC for banned compounds," Talanta, vol. 97, pp. 32-41, 2012.

[25] C. Yu and B. Hu, "C18-coated stir bar sorptive extraction combined with high performance liquid chromatographyelectrospray tandem mass spectrometry for the analysis of sulfonamides in milk and milk powder," Talanta, vol. 90, pp. 77-84, 2012.

[26] S. Bogialli, G. D’Ascenzo, A. Di Corcia, A. Laganà, and S. Nicolardi, "A simple and rapid assay based on hot water extraction and liquid chromatography-tandem mass spectrometry for monitoring quinolone residues in bovine milk," Food Chemistry, vol. 108, no. 1, pp. 354-360, 2008.

[27] S. Bogialli, G. D’Ascenzo, A. Di Corcia, A. Laganà, and G. Tramontana, "Simple assay for monitoring seven quinolone antibacterials in eggs: extraction with hot water and liquid chromatography coupled to tandem mass spectrometry," Journal of Chromatography A, vol. 1216, no. 5, pp. 794-800, 2009.

[28] R. W. Han, N. Zheng, Z. N. Yu et al., "Simultaneous determination of 38 veterinary antibiotic residues in raw milk by UPLC-MS/MS," Food Chemistry, vol. 181, pp. 119-126, 2015.

[29] A. V. Herrera-Herrera, J. Hernández-Borges, M. A. RodríguezDelgado, M. Herrero, and A. Cifuentes, "Determination of quinolone residues in infant and young children powdered milk combining solid-phase extraction and ultra-performance liquid chromatography-tandem mass spectrometry," Journal of Chromatography A, vol. 1218, no. 42, pp. 7608-7614, 2011.

[30] S. Herranz, M. C. Moreno-Bondi, and M. D. Marazuela, "Development of a new sample pretreatment procedure based on pressurized liquid extraction for the determination of fluoroquinolone residues in table eggs," Journal of Chromatography $A$, vol. 1140, no. 1-2, pp. 63-70, 2007.

[31] Z. Zhang, X. Li, S. Ding, H. Jiang, J. Shen, and X. Xia, "Multiresidue analysis of sulfonamides, quinolones, and tetracyclines in animal tissues by ultra-high performance liquid chromatography-tandem mass spectrometry," Food Chemistry, vol. 204, pp. 252-262, 2016.

[32] C. M. Karbiwnyk, L. E. Carr, S. B. Turnipseed, W. C. Andersen, and K. E. Miller, "Determination of quinolone residues in shrimp using liquid chromatography with fluorescence detection and residue confirmation by mass spectrometry," Analytica Chimica Acta, vol. 596, no. 2, pp. 257-263, 2007.

[33] W. C. Andersen, S. B. Turnipseed, J. E. Roybal, B. S, and J. E. Roybal, "Quantitative and confirmatory analyses of malachite green and leucomalachite green residues in fish and shrimp," Journal of Agricultural and Food Chemistry, vol. 54, no. 13, pp. 4517-4523, 2006.

[34] Y. Tao, D. Chen, X. Chao et al., "Simultaneous determination of malachite green, gentian violet and their leuco-metabolites in shrimp and salmon by liquid chromatography-tandem mass spectrometry with accelerated solvent extraction and auto solid-phase clean-up," Food Control, vol. 22, no. 8, pp. 1246-1252, 2011.

[35] L. Valle, C. Díaz, A. L. Zanocco, and P. Richter, "Determination of the sum of malachite green and leucomalachite green in salmon muscle by liquid chromatography-atmospheric pressure chemical ionisation-mass spectrometry," Journal of Chromatography A, vol. 1067, no. 1-2, pp. 101-105, 2005. 